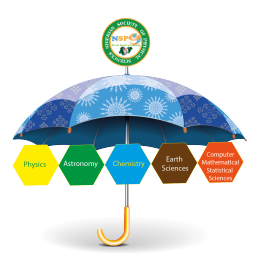

\title{
Energy distribution of an ion cloud in a quadrupole Penning Trap
}

\author{
B. M. Dyavappa* \\ Department of Physics, Government College for Women, Kolar, India.
}

\begin{abstract}
Ions are confined in Penning trap by the combination of electric and magnetic fields, as the electric field confines ions in the axial direction through an electric potential minimum and the magnetic field applied along the axis of the trap confines the ions in the radial direction. In the high temperature limit Coulomb interaction of ions can be neglected and the total energy is due to the electrostatic potential energy of the charge of ions and kinetic energy due to thermal energy. However, in the low temperature limit the trapping potential created by the DC voltage applied between the end cap and ring electrodes is cancelled by Coulomb interaction of ions and the total energy is mainly kinetic energy of ions. The probability density of energy distribution of ions along axial direction, in radial plane and the total probability density of energy distribution due to resulting motion of both axial and radial motion of ions under high temperature and low temperature limits in a Quadrupole Penning trap are presented here. These results reveal the energy properties of ion cloud and are useful to carry out accurate measurement experiments on single stored particle, antiparticles with energy related parameters, under high temperature and low temperature limits in a Quadrupole Penning trap.
\end{abstract}

Keywords: Quadrupole Penning trap, Probability of energy distribution function, Probability density of energy distribution function

Article History :

Received: 25 January 2020

Received in revised form: 09 March 2020

Accepted for publication: 20 March 2020

Published: 14 May 2020

(C)2020 Journal of the Nigerian Society of Physical Sciences. All rights reserved. Communicated by: B. J. Falaye

\section{Introduction}

Penning traps are used for high precision mass spectrometry, time of flight detection of ion cyclotron and magnetron resonances and for cooling of antiprotons, spectral intensity distribution of fermions [1], quantum information processing [2] etc. A thorough knowledge of different properties of stored ions like stability parameters, energy distribution, potential depths, space charge effects, anharmonicities etc. in the trap are essential. The Penning trap uses a time-independent, spatially homogeneous uniform magnetic field along the symmetry axis of the trap. The end-cap electrodes are biased with a positive DC voltage for the confinement of positive ions or negative DC voltage for the confinement of negative ions with respect to the

\footnotetext{
${ }^{*}$ Corresponding author tel. no: +919483113600

Email address: dyavappabm@gmail.com (B. M. Dyavappa)
}

ring electrode; hence both electric and magnetic fields are superposed to trap ions, as the resultant of quadrupolar potential in a small region of space (see Figure 1). The quadrupole Penning trap is made with three-electrodes; two are end-cap electrodes and a ring electrode. The equation of ring electrode is $\frac{r^{2}}{r_{0}^{2}}-\frac{z^{2}}{z_{0}^{2}}=+1$ and those of two similar end-cap electrodes is $\frac{r^{2}}{r_{0}^{2}}-\frac{z^{2}}{z_{0}^{2}}=-1$, where $r_{0}$ is the inner radius of the ring electrode in the radial plane and $z_{0}$ is half of the vertical distance between the two end-cap electrodes designed such that $r_{0}=\sqrt{2} z_{0}$. The trap potential is $[3,4]$

$V_{T}(r, z)=\frac{V_{0}}{r_{0}^{2}+2 z_{0}^{2}}\left(2 z^{2}-r^{2}\right)$

The potential well due to the electric field in the axial direction 


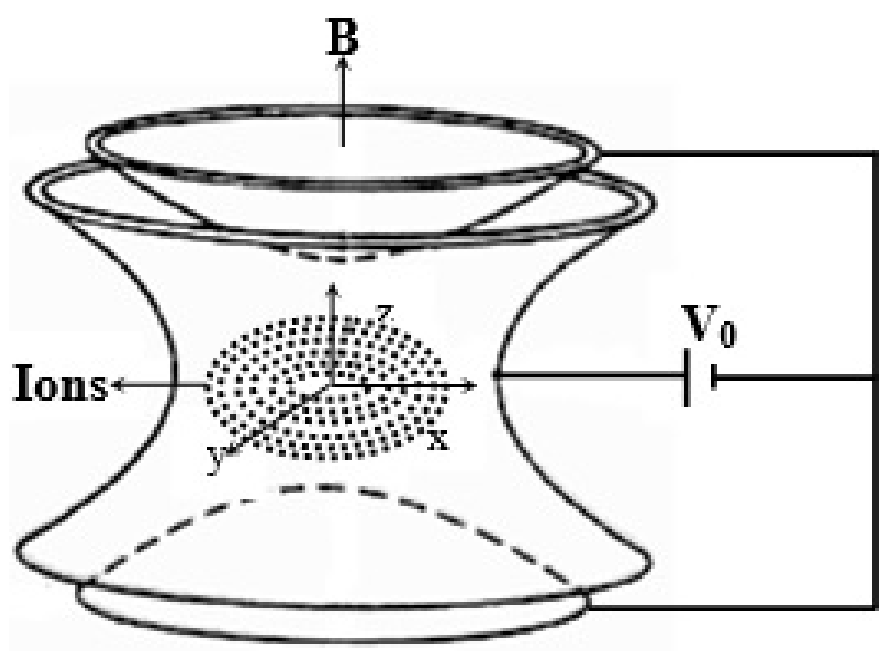

Figure 1: Quadrupole Penning trap with cloud of ions confined in trap space.

and radial plane is given by [5]

$V_{T}(r, z)=\frac{1}{4 \pi \epsilon_{0} r}\left[q e^{-\left(\frac{r}{\lambda_{D}}\right)}\right]+\frac{m d^{2} \omega_{z}^{2}}{4 q}$

where $\lambda_{D}=\left(\epsilon_{0} k_{B} T / n q^{2}\right)$ is Debye length, $r$ is the radial coordinate, $n$ is density of particles, $T$ is temperature and $q$ is the charge of ion. The plasma parameter $\left(N_{D}=4 \pi n \lambda_{D}^{3} / 3\right)$ gives the average number of particles found in the Debye sphere of plasma. Penning traps are used in high precision mass spectrometry $[6,7]$ for cooling antiprotons for measuring their gravitational acceleration [8]. Ions are confined first in a catching trap and then transferred to high precision trap to carry out measurements. Time of flight technique is used to detect ion cyclotron and magnetron resonances. A thorough knowledge of different properties of ions including energy distribution along axial direction, in radial plane and the total energy distribution is necessary to understand these processes.

\section{Theory}

We assume that the ion cloud is in thermal equilibrium through Coulomb interaction between ions. The axial component of angular momentum of the ions is conserved as the Penning trap is symmetric along its axis in the absence of external torques. This causes the rotation of the ion cloud as a whole [9] and the rotation in presence of magnetic field provides radial confining force to balance the electrostatic force of expansion. Therefore the rotation in magnetic field is equivalent to neutralization by opposite charge of ions and the distribution of magnetically confined ions in thermal equilibrium without rotation can be treated as ions confined and neutralized by a cylinder of opposite charge. The axial, pure cyclotron, reduced cyclotron and magnetron frequencies are given respectively by $[10,11,12$, 13]

$f_{z}=\frac{1}{2 \pi} \sqrt{\frac{4 e V_{0}}{m d^{2}}}, f_{c}=\frac{e B}{2 \pi m}, f_{c}^{\prime}=\frac{f_{c}+\sqrt{f_{c}^{2}-2 f_{z}^{2}}}{2}, f_{m}=\frac{f_{c}-\sqrt{f_{c}^{2}-2 f_{z}^{2}}}{2}$, $\therefore f_{m}<f_{\phi}<f_{c}-f_{m}$.

The probability of energy distribution function in thermal-equilibrium is [14]

$$
\begin{gathered}
d P\left(E_{r, \phi, z, P_{r}, P_{\phi}, P_{z}}\right)=\frac{2 \pi}{\left(\pi k_{B} T\right)^{\frac{3}{2}}} E^{\frac{1}{2}} \exp \left[-\left(\frac{E-\omega_{\phi} P_{\phi}}{k_{B} T}\right)\right] . \\
d r d \phi d z d P_{r} d P_{\phi} d P_{z}
\end{gathered}
$$

where $\omega_{\phi}$ is the rotational frequency of ion cloud as a whole determined by total angular momentum and energy determined by the temperature of the ion cloud.

Energy of single ion is [14]

$$
\begin{gathered}
E=\frac{1}{2 m}\left[P_{r}^{2}+\left(\frac{P_{\phi}}{r}-\frac{q B r}{2 c}\right)^{2}+P_{z}^{2}\right]+ \\
q\left[V_{T}(r, z)+V_{q}(r, z)\right]
\end{gathered}
$$

The momentums in radial plane, azimuthal and axial directions are [14]

$$
\left.\begin{array}{r}
P_{r}=m v_{r}=m \frac{d r}{d t}, \\
P_{\phi}=m r^{2} \frac{d \phi}{d t}+\frac{q B}{2 c} r^{2}, \\
=m r v_{\phi}-\frac{\omega_{c}}{2} m r^{2}, \\
P_{z}=m \frac{d z}{d t}=m v_{z}
\end{array}\right\}
$$

Coulomb interaction potential of ions is [14]

$V_{q}(r, z)=\int_{0}^{\infty} \frac{n q\left(r^{\prime}, z^{\prime}\right)}{\left|r-r^{\prime}\right|} r^{\prime} d r^{\prime} d \phi^{\prime} d z^{\prime}$

\section{Energy distribution of an ion cloud at the high tempera- ture limit}

The Penning trap is designed with its dimension to be $20 \mathrm{~mm}$ and magnetic field applied is $6 T$, when the DC voltage applied is $3000 \mathrm{~V}$ for an ion cloud density of $10^{14} \mathrm{~m}^{-3}$ with Debye length $2.4 \times 10^{-3} \mathrm{~m}$, the energy and temperature of ions at high temperature limit considered are $10 \mathrm{eV}, 116045.05 \mathrm{~K}$ respectively. In high temperature limit [14]

$q V_{q}(r, z) \ll k_{B} T \ll q V_{T}(r, z)+\frac{m}{2} \omega_{\phi}\left(\omega_{c}-\omega_{\phi}\right) r^{2}$

If we neglect Coulomb interaction $V_{q}(r, z)$ then the probability of energy distribution function is [14]

$$
\begin{array}{r}
d P\left(E_{\left.r, \phi, z, P_{r}, P_{\phi}, P_{z}\right) \quad=} A \exp \left[-\left(\frac{E_{z}+E_{r}^{\prime}}{k_{B} T}\right)\right] .\right. \\
d r d \phi d z d P_{r} d P_{\phi} d P_{z}
\end{array}
$$

The energy along Z-direction is [14]

$E_{z}=\frac{1}{2 m} P_{z}^{2}+k_{z} z^{2}$

The energy in the radial plane is [14]

$$
\begin{gathered}
E_{r}=\frac{1}{2 m}\left(P_{r}^{2}+\frac{P_{\phi}^{2}}{r^{2}}\right)+\left(\frac{m \omega_{c}^{2}}{8}+\frac{q V_{0}}{d^{2}}\right) r^{2}+ \\
\frac{\omega_{c}}{2} P_{\phi}
\end{gathered}
$$




$$
\begin{array}{cc}
E_{r}^{\prime}= & E_{r}-\omega_{\phi} P_{\phi} \\
= & \frac{1}{2 m}\left(P_{r}^{2}+\frac{P_{\phi}^{2}}{r^{2}}\right)+\left(\frac{m \omega_{c}^{2}}{8}+\frac{q V_{0}}{d^{2}}\right) r^{2}+ \\
& \left(\frac{\omega_{c}}{2}-\omega_{\phi}\right) P_{\phi}
\end{array}
$$

The energy distribution in Z-direction is [14]

$d P\left(E_{z, P_{z}}\right)=\operatorname{Bexp}\left[-\left(\frac{E_{z}}{k_{B} T}\right)\right] d z d P_{z}$

The probability density of energy distribution in Z-direction is [14]

$\rho_{z}\left(E_{z}\right)=\frac{1}{k_{B} T} \exp \left(-\frac{E_{z}}{k_{B} T}\right)$

The average energy in Z-direction is [14]

$$
\left\langle E_{z}\right\rangle=\int_{0}^{\infty} E_{z} \rho_{z}\left(E_{z}\right) d E_{z}=k_{B} T
$$

The energy distribution in the radial direction is [14]

$$
\begin{aligned}
& d P\left(E_{r, \phi, P_{r}, P_{\phi}}\right)=C \exp \left(-\frac{E_{r}^{\prime}}{k_{B} T}\right) d r d \phi d P_{r} d P_{\phi} \\
& d P\left(E_{r}^{\prime}\right)=\left(\frac{1}{k_{B} T}\right)^{2} \exp \left(-\frac{E_{r}^{\prime}}{k_{B} T}\right) E_{r}^{\prime} d E_{r}^{\prime}
\end{aligned}
$$

The probability density of energy distribution in the high temperature limit with $\omega_{\phi} \ll \frac{\omega_{c}}{2}$ is [14]

$$
\begin{gathered}
\rho_{r}\left(E_{r}^{\prime}\right)=\left(\frac{1}{k_{B} T}\right)^{2} \exp \left(-\frac{E_{r}^{\prime}}{k_{B} T}\right) E_{r}^{\prime} \\
\rho_{r}\left(E_{r}\right)=\left(\frac{1}{k_{B} T}\right)^{2} \exp \left(-\frac{E_{r}}{k_{B} T}\right) E_{r} \\
\left\{\because E_{r} \approx E_{r}^{\prime}\right\}
\end{gathered}
$$

The average energy in the radial plane is [14]

$$
\left\langle E_{r}\right\rangle=\int_{0}^{\infty} E_{r} \rho_{r}\left(E_{r}\right) d E_{r}=2 k_{B} T
$$

The total probability of energy distribution is [14]

$$
\begin{aligned}
d P(E) \quad & =\rho_{z}\left(E_{z}\right) \rho_{r}\left(E_{r}\right) d E_{z} d E_{r} \\
= & \left(\frac{1}{k_{B} T}\right)^{3} \exp \left(-\frac{E}{k_{B} T}\right) E_{r} d E_{z} d E_{r}
\end{aligned}
$$

The total probability density of energy distribution is [14]

$\rho\left(\frac{E}{k_{B} T}\right)=\frac{1}{2}\left(\frac{1}{k_{B} T}\right)^{3} \exp \left(-\frac{E}{k_{B} T}\right) E^{2}$

The total average energy is [14]

$$
\begin{gathered}
\langle E\rangle=\int_{0}^{\infty} E \rho(E) d E=\left\langle E_{z}\right\rangle+\left\langle E_{r}\right\rangle \\
=k_{B} T+2 k_{B} T=3 k_{B} T
\end{gathered}
$$

The total energy is [14]

$$
E=E_{z}+E_{r}
$$

The probability density of energy distribution in axial direction increases sharply up to $24.3559 \times 10^{16}$ at $E=1.3375 k_{B} T$, decreases abruptly and remains almost a constant for high temperature limit as shown in Figure 2(a). The probability density of energy distribution in the radial plane increases sharply up to $34.4544 \times 10^{16}$ at $E=2.11487 k_{B} T$, decreases abruptly and remains almost a constant for the high temperature limit as shown in the Figure 2(b). The total probability density of energy distribution increases sharply up to $42.5566 \times 10^{16}$ at $E=3.0728 k_{B} T$ , decreases abruptly and remains almost a constant for the high temperature limit as shown in the Figure 2(c). The axial, radial and total probability densities of energy distribution increase sharply up to $24.3559 \times 10^{16}, 34.4544 \times 10^{16}, 42.5566 \times 10^{16}$ at $E=1.3375 k_{B} T, 2.1149 k_{B} T, 3.0728 k_{B} T$ respectively, decrease abruptly and remain almost constant as $k_{r}^{\prime} r^{2} / k_{B} T \approx 5.4 \times 10^{2}$ and $k_{B} T / q V_{q}(r, z) \approx\left(\lambda_{D} / r\right)^{2} \approx 6$ for the high temperature limit as shown in Figure 2(d). The axial probability density of energy distribution is less than the radial probability density of energy distribution, which in turn less than the total probability density of energy distribution. This analysis helps to design trap that consists of appropriate structure trap electrodes to minimize anharmonicities to improve storage time.

\section{Energy distribution of an ion cloud at the low tempera- ture limit}

In a Penning trap of dimension $10 \mathrm{~mm}$, magnetic field $2 T$, when the DC voltage applied is $10 V$ for an ion cloud density $2 \times$ $10^{14} \mathrm{~m}^{-3}$ and Debye length $10^{-5} \mathrm{~m}$, the energy and temperature of ions at low temperature limit considered are $3.6 \times 10^{-4} \mathrm{eV}$, $4.2 \mathrm{~K}$ respectively. In the low temperature limit [14]

$q V_{q}(r, z) \gg k_{B} T$

$q V_{T}(r, z)+q V_{q}(r, z)+\frac{m}{2} \omega_{\phi}\left(\omega_{c}-\omega_{\phi}\right) r^{2}=0$

If we neglect Coulomb interaction potential $V_{q}(r, z)$ then probability of energy distribution distribution function is [14]

$$
\begin{array}{r}
d P\left(E_{P_{r}, P_{\phi}, P_{z}}\right)=A^{\prime} \exp \left[-\left(\frac{E_{z}+E_{r}^{\prime}}{k_{B} T}\right)\right] . \\
d P_{r} d\left(\frac{P_{\phi}}{r}\right) d P_{z}
\end{array}
$$

The energy in Z-direction is [14]

$E_{z}=\frac{1}{2 m} P_{z}^{2}$

The energy in the radial plane is [14]

$$
\begin{array}{r}
E_{r}=\frac{1}{2 m}\left(P_{r}^{2}+\frac{P_{\phi}^{2}}{r^{2}}\right)+\left(\frac{m \omega_{c}^{2}}{8}\right) r^{2}+\frac{\omega_{c}}{2} P_{\phi} \\
E_{r}^{\prime}=E_{r}-\omega_{\phi} P_{\phi} \quad=\frac{1}{2 m}\left(P_{r}^{2}+\frac{P_{\phi}^{2}}{r^{2}}\right)+ \\
\left(\frac{m \omega_{c}^{2}}{8}\right) r^{2}+\left(\frac{\omega_{c}}{2}-\omega_{\phi}\right) P_{\phi}
\end{array}
$$


Table 1: The values of axial, radial and total probability density of energy distribution in the high temperature limit.

\begin{tabular}{lcccccc}
\hline$\frac{E}{k_{B} T}$ & $e^{-\frac{E}{k_{B} T}}$ & $T(K)$ & $k_{B} T\left(10^{-19} J\right)$ & $\rho_{z}\left(\frac{E_{z}}{k_{B} T}\right)\left(10^{16}\right)$ & $\rho_{r}\left(\frac{E_{r}}{k_{B} T}\right)\left(10^{16}\right)$ & $\rho\left(\frac{E}{k_{B} T}\right)\left(10^{16}\right)$ \\
\hline 0 & 1 & $\infty$ & $\infty$ & 0 & 0 & 0 \\
1 & 0.367879 & 115942 & 15.999996 & 22.9924 & 22.9924 & 11.4962 \\
2 & 0.135335 & 57971 & 7.999998 & 16.9169 & 33.8338 & 33.8338 \\
3 & 0.049787 & 38647.3 & 5.3333274 & 9.33507 & 28.0052 & 42.0078 \\
4 & 0.018316 & 28985.5 & 3.999999 & 4.579 & 18.316 & 36.632 \\
5 & 0.006738 & 23188.4 & 3.1999992 & 2.1056 & 10.528 & 26.32 \\
6 & 0.00247875 & 19323.7 & 2.6666706 & 0.92953 & 5.57718 & 16.73154 \\
7 & 0.00091188 & 16563.1 & 2.2857078 & 0.39895 & 2.79265 & 9.774275 \\
8 & 0.00033546 & 14492.75 & 1.9999995 & 0.16773 & 1.34184 & 5.36736 \\
9 & 0.00012341 & 12882.4 & 1.7777712 & 0.06942 & 0.62478 & 2.8115 \\
10 & 0.0000454 & 11594.2 & 1.5999996 & 0.028375 & 0.28375 & 1.41875 \\
\hline
\end{tabular}

Table 2: The values of axial, radial and total probability density of energy distribution in the low temperature limit.

\begin{tabular}{lcccccc}
\hline$\frac{E}{k_{B} T}$ & $e^{-\frac{E}{k_{B} T}}$ & $T(K)$ & $k_{B} T\left(10^{-23} J\right)$ & $\rho_{z}\left(\frac{E_{z}}{k_{B} T}\right)\left(10^{20}\right)$ & $\rho_{r}\left(\frac{E_{r}}{k_{B} T}\right)\left(10^{20}\right)$ & $\rho\left(\frac{E}{k_{B} T}\right)\left(10^{20}\right)$ \\
\hline 0 & 1 & $\infty$ & $\infty$ & 0 & 0 & 0 \\
1 & 0.367879 & 4.2 & 5.796 & 35.8098 & 63.471187 & 71.6195545 \\
2 & 0.135335 & 2.1 & 2.898 & 18.63038 & 46.699448 & 74.521537 \\
3 & 0.049787 & 1.4 & 1.932 & 8.39408 & 25.7696687 & 50.3644996 \\
4 & 0.018316 & 1.05 & 1.449 & 5.166848 & 12.640442 & 28.5264222 \\
5 & 0.006738 & 0.84 & 1.1592 & 1.4666034 & 5.812693 & 14.666034 \\
6 & 0.00247875 & 0.7 & 0.966 & 0.591024 & 2.565994 & 7.0922868 \\
7 & 0.00091188 & 0.6 & 0.828 & 0.234846 & 1.101304 & 3.2878457 \\
8 & 0.00033546 & 0.525 & 0.7245 & 0.09235968 & 0.4630228 & 1.4777549 \\
9 & 0.00012341 & 0.4667 & 0.644 & 0.01325787 & 0.1916304 & 0.6486954 \\
10 & 0.0000454 & 0.42 & 0.5796 & 0.013975 & 0.07832988 & 0.2795
\end{tabular}

The probability of energy distribution in Z-direction is [14]

$d P\left(E_{P_{z}}\right)=B^{\prime} \exp \left[-\left(\frac{E_{z}}{k_{B} T}\right)\right] d P_{z}$

The probability density of energy distribution in Z-direction is [14]

$\rho_{z}\left(\frac{E_{z}}{k_{B} T}\right)=\sqrt{\frac{1}{\pi k_{B} T}}\left[\exp \left(-\frac{E_{z}}{k_{B} T}\right)\right] \frac{1}{\sqrt{E_{z}}}$

The average energy in Z-direction is [14]

$\left\langle E_{z}\right\rangle=\int_{0}^{\infty} E_{z} \rho_{z}\left(E_{z}\right) d E_{z}=\frac{1}{2} k_{B} T$

The probability of energy distribution in the radial plane is [14]

$d P\left(E_{P_{r}, P_{\phi}}\right)=C^{\prime} \exp \left(-\frac{E_{r}^{\prime}}{k_{B} T}\right) d P_{r} d P_{\phi}$

$d P\left(\frac{E_{r}^{\prime}}{k_{B} T}\right)=\left(\frac{1}{k_{B} T}\right) \exp \left(-\frac{E_{r}^{\prime}}{k_{B} T}\right) d E_{r}^{\prime}$

The probability density of energy distribution in radial plane in the low temperature limit with $\omega_{\phi} \gg \omega_{c} / 2$ is [14]

$\rho_{r}\left(\frac{E_{r}^{\prime}}{k_{B} T}\right)=\left(\frac{1}{k_{B} T}\right) \exp \left(-\frac{E_{r}^{\prime}}{k_{B} T}\right)$

$$
\begin{array}{r}
\rho_{r}\left(\frac{E_{r}}{k_{B} T}\right)=\left(\frac{1}{k_{B} T}\right) \exp \left(-\frac{E_{r}}{k_{B} T}\right) \\
\left\{\because E_{r} \approx E_{r}^{\prime}\right\}
\end{array}
$$

The average energy in radial plane is [14]

$\left\langle E_{r}\right\rangle=\int_{0}^{\infty} E_{r} \rho_{r}\left(E_{r}\right) d E_{r}=k_{B} T$

The total probability density of energy distribution is [14]

$\rho(E)=\left(\frac{4}{\pi}\right)^{\frac{1}{2}}\left(\frac{1}{k_{B} T}\right)^{\frac{3}{2}} \exp \left(-\frac{E}{k_{B} T}\right) \sqrt{E}$

The total average energy is [14]

$\langle E\rangle=\left\langle E_{z}\right\rangle+\left\langle E_{r}\right\rangle=\frac{1}{2} k_{B} T+k_{B} T=\frac{3}{2} k_{B} T$

The probability density of energy distribution in axial direction increases sharply up to $36.6166 \times 10^{20}$ at $E=1.1466 k_{B}$, decreases abruptly and remains almost a constant for the low temperature limit as shown in Figure 3(a). The total probability density of energy distribution in the radial plane increases sharply up to $63.59 \times 10^{20}$ at $E=0.99 k_{B} T$, decreases abruptly and remains almost a constant for the low temperature limit as shown in the Figure 3(b). The total probability density of energy distribution increases sharply up to $83.39 \times 10^{20}$ at $E=$ $1.674 k_{B} T$, decreases abruptly and remains almost a constant 

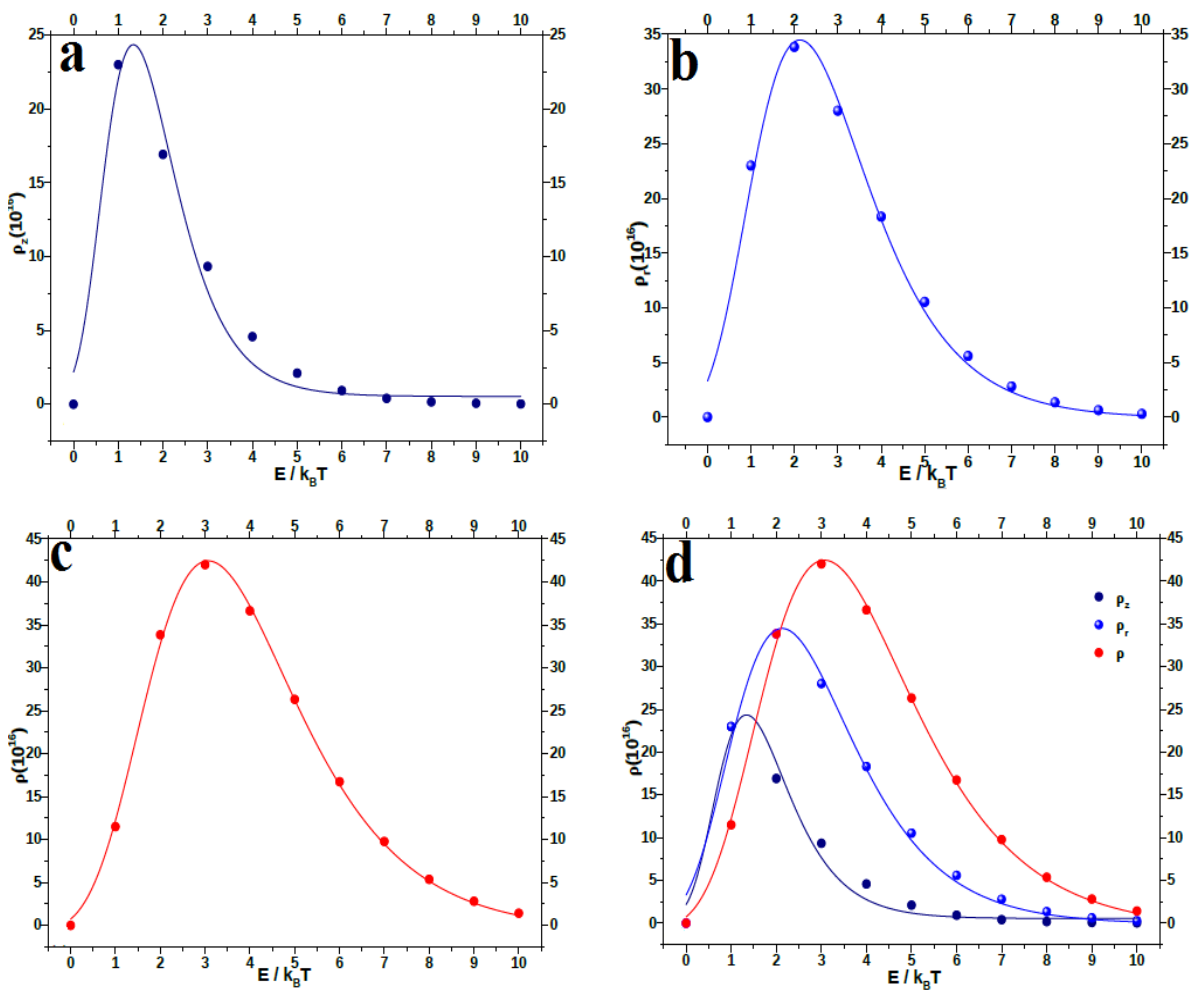

Figure 2: (a) The probability density of energy distribution in axial direction in the high temperature limit; (b) The probability density of energy distribution in the radial plane in the high temperature limit; (c) The total probability density of energy distribution in the high temperature limit; (d) The axial, radial and total probability densities of energy distribution in the high temperature limit.
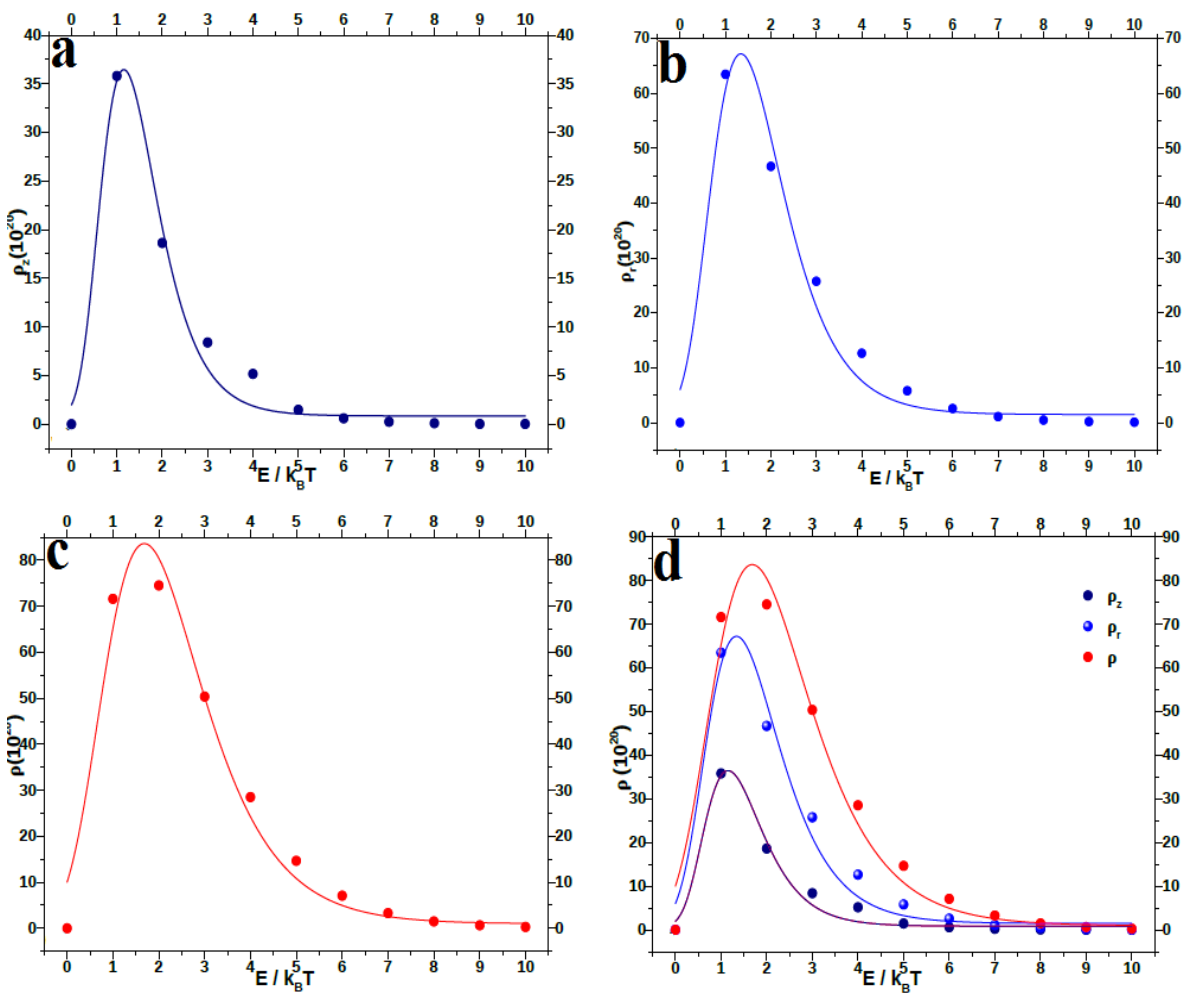

Figure 3: (a) The probability density of energy distribution in axial direction in the low temperature limit; (b) The probability density of energy distribution in the radial plane in the low temperature limit; (c) The total probability density of energy distribution in the low temperature limit; (d) The axial, radial and total probability density of energy distribution in the low temperature limit. 
for the low temperature limit as shown in the Figure 3(c). The axial, radial and total probability densities of energy distribution increase sharply up to $36.6166 \times 10^{20}, 63.59 \times 10^{20}, 83.39 \times$ $10^{20}$ at $E=1.1466 k_{B} T, 0.99 k_{B} T, 1.674 k_{B} T$ respectively, decrease abruptly, remain almost constant as $\lambda_{D} / r \ll 1, \lambda_{D} / z \ll 1$ for the low temperature limit as shown in the Figure 3(d). The axial probability density of energy distribution is less than the radial probability density of energy distribution, which in turn less than the total probability density of energy distribution.

The total probability densities of energy distributions increase sharply up to $42.5566 \times 10^{16}, 83.39 \times 10^{20}$ at $E=$ $3.0728 k_{B} T, 1.674 k_{B} T$ decrease abruptly and remain almost constants under high and low temperature limits respectively as shown in the Figure 4.

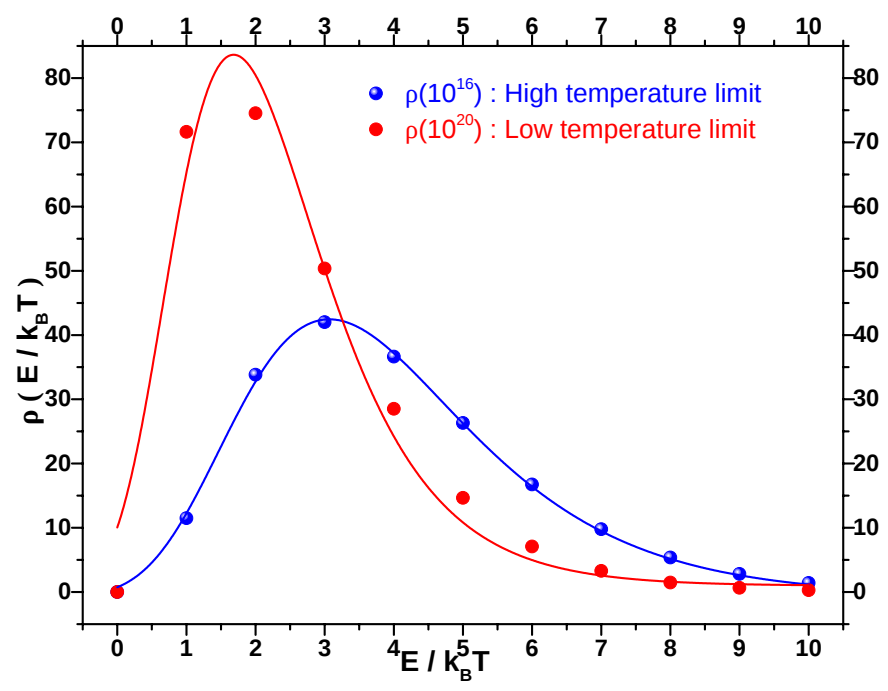

Figure 4: The total probability density of energy distribution when $f_{\phi} \ll$ $f_{c} / 2$ in the high temperature and low temperature limits.

\section{Conclusion}

The probability density of energy distribution of ions along axial direction and in radial plane together results total probability density of energy distribution under high temperature limit and similarly at low temperature limit measured in a Quadrupole Penning trap. The total probability density of energy distribution in the low temperature limit is much sharper and greater than that at the high temperature limit. These results reveal the energy properties of an ion cloud and are useful to design and carry out experiments on stored single particle, antiparticles with energy related parameters, under high temperature and low temperature limits in Quadrupole Penning trap.

\section{Acknowledgments}

We thank the referees for the positive enlightening comments and suggestions, which have greatly helped us in making improvements to this paper. The author also acknowledges Dr. Satyajith K. T., Assistant Professor of Physics, Amritha Vishwa Vidhya Peetam, Coimbatur, Tamil Nadu, India, for important suggestion on energy distribution function.

\section{References}

[1] S. K. Ghosh "spectral intensity distribution of trapped fermions", Pramana journal of physics 85 (2015) 616.

[2] Dong et al. "A review of silicon microfabricated ion traps for quantum information processing, Micro and Nano Systems", Letters (2015).

[3] F. G. Major, V. N. Gheorghe \& G. Werth, "Charged Particle Traps, Physics and techniques of charged particle confinement", Springer (2005).

[4] Pradip K.Ghosh Ion Traps, Clarendon Press, Oxford (1995) 72.

[5] U. Shumacher "Basics of plasma physics", Lect.Notes Phys. 670 (2005) 20.

[6] C. Gerz, D. Wilsdorf \& G. Werth, "A high precision Penning trap mass spectrometer", Nuclear Instruments and Methods in Physics Research Section B: Beam Interactions with Materials and Atoms. Z.Phys. D 47 (1990) 453.

[7] S. Becker, G. Bollen, F. Kern, H. J. Kluge, R. B. Moore, G. Savard, L. Schweikhard, H. Stolzenberg \& ISOLDE collaboration, "Mass measurements of very high accuracy by time-of-flight ion cyclotron resonance of ions injected into a penning trap", Int. J. Mass Spectrom. Ion Processes 99 (1990) 53.

[8] N. Beverini, B. E. Bonner \& J. H. Billen, "A measurement of the gravitational acceleration of the antiprotons", Los Alamos National Laboratory Document LA-UR-86-260 (1986).

[9] T. M. O'Neil \& C. F. Driscoll, "Transport to thermal equilibrium of a pure electron plasma", The Physics of Fluids 22 (1979) 266.

[10] D. Datar, B. M. Dyavappa, B. L. Mahesh, K. T. Satyajith \& S. Ananthamurthy, "Energy distribution of electrons under axial motion in a quadrupole Penning trap", Can. J. Phys 94 (2016) 1249.

[11] B. M. Dyavappa, "Velocity distribution of electrons along the symmetry axis of Quadrupole Penning trap", Discovery 56 (2020) 141.

[12] B. M. Dyavappa, D. D. Prakash \& S. Ananthamurthy "Dependence of the confinement time of an electron plasma on the magnetic field in a quadrupole Penning trap", EPJ Techniques and Instrumentation 4 (2017) DOI: 10.1140/epjti/s40485-017-0039-4.

[13] B. M. Dyavappa Spectroscopy of non-neutral plasmas in ion traps Ph.D. thesis submitted to Bangalore University (2017).

[14] G. Z. Li \& G. Werth, "Energy distribution of ions in Penning trap", Int. J. Mass Spectro. Ion Processes 121 (1992) 75. 